\title{
Lipoprotein metabolism in an apoB-80 familial hypobetalipoproteinemia heterozygote
}

Amanda J. Hooper, ${ }^{\mathrm{a}, \mathrm{b}, \mathrm{c}}$, Ken Robertson ${ }^{\mathrm{a}, \mathrm{c}}$, Danie Champain ${ }^{\mathrm{b}}$, Jianmin Hua ${ }^{\mathrm{d}}$, Swithin Song ${ }^{\mathrm{d}}$, Klaus G.

Parhofer $^{\mathrm{e}}$, P. Hugh R. Barrett ${ }^{\mathrm{b}}$, Frank M. van Bockxmeer ${ }^{\mathrm{a}, \mathrm{f}}$, John R. Burnett ${ }^{\mathrm{a}, \mathrm{b}}$

${ }^{a}$ Department of Clinical Biochemistry, PathWest Laboratory Medicine WA, Royal Perth Hospital and Fiona Stanley Hospital Network, Perth, Australia;

${ }^{\mathrm{b}}$ School of Medicine and Pharmacology, University of Western Australia, Crawley, Australia;

'School of Pathology and Laboratory Medicine, University of Western Australia, Crawley, Australia;

${ }^{\mathrm{d}}$ Department of Radiology, Royal Perth Hospital, Perth, Australia;

'Medical Department II, Grosshadern, University of Munich, Munich, Germany;

${ }^{\mathrm{f}}$ School of Surgery, University of Western Australia, Crawley, Australia

Corresponding author: Dr. John R. Burnett, Department of Clinical Biochemistry, PathWest

Laboratory Medicine WA, Royal Perth Hospital, Wellington Street, GPO Box X2213, Perth, WA 6847, Australia.

Phone: +61-8-9224-3121

Fax: +61-8-9224-1789

Email: john.burnett@health.wa.gov.au

Number of words (main text): 1323

Number of figures/tables: 1

Key words: Familial hypobetalipoproteinemia $\square$ apolipoprotein B $\mathbf{a}$ lipoprotein $\mathbf{~ k i n e t i c s ~}$ metabolism — compartmental modeling

Abbreviations: apo, apolipoprotein; FCR, fractional catabolic rate; FHBL, familial hypobetalipoproteinemia; IDL, intermediate density lipoprotein; LDL, low density lipoprotein; VLDL, very low density lipoprotein 


\begin{abstract}
Objective: Familial hypobetalipoproteinemia (FHBL) is characterized by mutations in $A P O B$, the majority of these causing protein truncations, and low plasma levels of apolipoprotein (apo) B. The hypobetalipoproteinemia may be due to enhanced clearance and possibly reduced production of apoBcontaining lipoproteins; the mechanism may depend on the length of the apoB truncation. We studied fasting lipoprotein metabolism in an FHBL subject heterozygous for a mutation causing a truncated apoB, apoB-80.
\end{abstract}

Design and Methods: Very low density lipoprotein (VLDL)-, intermediate density lipoprotein (IDL), and low density lipoprotein (LDL)-apoB kinetics were determined in the fasting state using stable isotope methods and compartmental modeling.

Results: Compared with lean normolipidemic controls the apoB-80 FHBL subject had an elevated VLDL-apoB fractional catabolic rate and lower LDL production. ApoB production rates and IDLand LDL-apoB fractional catabolic rates were not different.

Conclusion: FHBL subjects heterozygous for a mutation truncating apoB to $80 \%$ of full-length are able to produce VLDL-apoB normally, but have rapid clearance of these particles, resulting in low levels of circulating apoB. 


\section{Introduction}

Hepatic steatosis is common with a population prevalence of up to $30 \%$. The main causes of fatty liver are non-alcoholic fatty liver disease and alcoholic liver disease. Inborn errors of metabolism are less common causes of hepatic steatosis, which may arise due to defects in lipid metabolism or hepatic triglyceride export, fatty acid oxidation, or insulin signaling [1].

Familial hypobetalipoproteinemia (FHBL) is a codominant disorder of lipoprotein metabolism caused by mutations in the $A P O B$ gene and characterized by low levels of LDLcholesterol and apolipoprotein (apo) B in plasma [2-4]. ApoB is essential for the formation of the triglyceride-rich lipoproteins; apoB-48 forms chylomicrons from enterocytes and full-length apoB100 forms very low density lipoproteins (VLDL) from hepatocytes. ApoB-100 remains an integral structural component of the lipoprotein throughout the VLDL-IDL-LDL cascade, and acts as a ligand for clearance by the LDL-receptor. Individuals carrying a single FHBL APOB mutation have apoB concentrations about $1 / 4$ to $1 / 3$ of normal and are likely to have fatty liver. FHBL $A P O B$ mutation homozygotes have very low or absent plasma apoB and may be asymptomatic or have gastrointestinal and neurologic dysfunction [2,3].

Truncations in apoB as short as 5\% (apoB-5) and as long as 89\% (apoB-89) of full length apoB-100 can cause FHBL $[5,6]$. However, only truncations shorter than $~$ apoB-30 are found in plasma. A linear relationship has been observed between the length of the truncated apoB and its secretion rate in heterozygous FHBL [7].

We previously described a case of FHBL due to apoB-80 [8]. In brief, the patient presented age 32 years with marked hypocholesterolemia (LDL-cholesterol of $0.4 \mathrm{mmol} / \mathrm{L}$ and apoB of 0.13 $\mathrm{g} / \mathrm{L})$ together with biochemical and histological changes consistent with hepatic steatosis. An immunoblot of plasma for apoB showed the presence of a truncated apoB species, with the subject confirmed to be heterozygous for the apoB-80.5 (c.11040T $>\mathrm{G})$ mutation by DNA sequencing. The magnitude of hypocholesterolemia was greater than would have been expected for the degree of apoB truncation.

In FHBL heterozygotes, the mechanism for hepatic steatosis and reduced plasma apoB concentrations may depend on the type of $A P O B$ mutation and length of apoB produced by the mutant allele. We aimed to examine the effect of heterozygous apoB-80 FHBL on fasting lipoprotein metabolism. 


\section{Methods}

\subsection{Participants}

Stable isotope studies were performed on the apoB-80 FHBL heterozygote, and four healthy, normolipidemic controls from a previous study [9]. All subjects provided written consent and the study was approved by the Royal Perth Hospital Human Ethics Committee (EC 2006/078).

\subsection{Liver fat content}

Single voxel proton magnetic resonance spectroscopy (MRS) of the liver was performed on a Sonata 1.5T (Siemens). Sequences and quantification procedures for liver fat content were as described [10].

\subsection{Stable isotope studies}

Subjects were studied semi-recumbent after a $12 \mathrm{~h}$ overnight fast and allowed water only for the initial $16 \mathrm{~h}$ of the study. A single bolus of $\mathrm{D}_{3}$-leucine $(1 \mathrm{mg} / \mathrm{kg})$ was administered intravenously within a 2 min period into an antecubital vein via a Teflon cannula. Blood samples were taken at baseline and at 15, 30 and $45 \mathrm{~min}$, and 1, 2, 4, 5, 6, 8, 10 and $16 \mathrm{~h}$ after isotope injection. Additional blood samples were collected in the morning on the four following days after a $12 \mathrm{~h}$ fast.

Isolation and measurement of isotopic enrichment of apoB, and biochemical analyses of lipoprotein subfractions and plasma were performed as previously described [10].

SAAM II (The Epsilon Group, Charlottesville, VA) was used to model the VLDL, IDL and LDL apoB tracer data. The details and assumptions of the model were as described [9]. The fractional catabolic rates (FCR) of VLDL, IDL and LDL-apoB were derived from the model parameters giving the best fit. The corresponding production rates were calculated as the product of FCR and pool size, which equals the plasma concentration multiplied by plasma volume ( $4.5 \%$ of body weight).

\section{Results}

The heterozygous apoB-80 FHBL subject had a low fasting total cholesterol of $2.1 \mathrm{mmol} / \mathrm{L}$ with normal HDL-cholesterol $(1.3 \mathrm{mmol} / \mathrm{L})$, and very-low LDL-cholesterol $(0.6 \mathrm{mmol} / \mathrm{L})$ and apoB $(0.15$ $\mathrm{g} / \mathrm{L}$ ). His hepatic fat content as determined by liver MRS was 39\%. Noninvasive staging of the liver using Hepascore and transient elastography showed no evidence of advanced fibrosis.

Fasting apoB kinetic parameters were compared with lean normolipidemic controls (Table 1). While apoB production rates and IDL- and LDL-apoB FCRs were not different between the apoB-80 FHBL subject and controls, the apoB-80 FHBL subject had an elevated VLDL-apoB FCR, and lower LDL production. 


\section{Discussion}

The apoB-80 heterozygous FHBL subject can produce normal apoB-48 from both mutant and wildtype alleles in the intestine, and apoB-80 and apoB-100 in the liver. Heterozygous FHBL subjects with apoB truncations shorter than apoB-48, and therefore only a single fully-functional apoB-48 allele, have decreased postprandial triglyceride-rich lipoprotein production, but normal clearance [11]. In contrast, normal postprandial responses have been observed for apoB truncations longer than apoB48 [12].

Our stable isotope kinetic study showed that the production of VLDL-apoB in the apoB-80 FHBL heterozygote was normal, but the apoB concentration was very-low due to a high VLDL-apoB FCR. The low production rate and high-normal FCR of LDL-apoB results in an LDL concentration that is very low indicating that the VLDL produced is cleared directly, rather than be converted to IDL and LDL particles; consistent with apoB-89 FHBL heterozygotes who had normal apoB-100 production with enhanced clearance [13]. FHBL heterozygotes carrying the apoB L343V missense mutation showed normal VLDL-apoB production and higher FCR compared with lean control subjects [10].

Truncated forms of apoB are produced at lower rates than apoB-100 with secretion reduced by about $1.4 \%$ for each $1 \%$ of apoB truncated [7]. This suggests that in order to account for the higher VLDL-apoB production rate in apoB-80 FHBL compared with controls, apoB expression, particularly of the normal allele, is upregulated. For 'long' apoB truncations such as apoB-75 and apoB-89 that contain the LDL-receptor-binding domain, the clearance of the truncated species is faster than the clearance of apoB-100 $[13,14]$. Of interest, apoB-80 behaves more similarly to apoB89 ; in contrast apoB-75 is associated with reduced apoB production in addition to increased catabolism [14].

In summary, we have presented the case of an apoB-80 FHBL heterozygous subject with marked hepatic steatosis who was able to produce VLDL-apoB normally, but had rapid clearance of these particles, resulting in the observed very-low levels of circulating apoB.

\section{Acknowledgements}

This work was supported by National Health and Medical Research Council Project Grant 1010133 (to AJH, JRB and FMvB) and a Practitioner Fellowship from the Royal Perth Hospital Medical Research Foundation (to JRB). PHRB is a NHMRC Senior Research Fellow.

\section{Disclosure/Conflict of Interest}

The authors declare no conflict of interest.

\section{Author contributions}


AJH, PHRB, FMvB, and JRB designed the study. All authors participated in acquisition, analysis or interpretation of the data, in the drafting of the article or critical revision and in final approval of the version to be published.

\section{References}

[1] Hooper AJ, Adams LA, Burnett JR. Genetic determinants of hepatic steatosis in man. J Lipid Res 2011; 52:593-617.

[2] Welty FK. Hypobetalipoproteinemia and abetalipoproteinemia. Curr Opin Lipidol 2014; 25:161-168.

[3] Hooper AJ, Burnett JR. Update on primary hypobetalipoproteinemia. Curr Atheroscler Rep 2014; $16: 423$.

[4] Tarugi P, Averna M. Hypobetalipoproteinemia: genetics, biochemistry, and clinical spectrum. Adv Clin Chem 2011; 54:81-107.

[5] Talmud P, King-Underwood L, Krul E, Schonfeld G, Humphries S. The molecular basis of truncated forms of apolipoprotein B in a kindred with compound heterozygous hypobetalipoproteinemia. J Lipid Res 1989; 30:1773-1779.

[6] Yue P, Isley WL, Harris WS, Rosipal S, Akin CD, Schonfeld G. Genetic variants of ApoE account for variability of plasma low-density lipoprotein and apolipoprotein B levels in FHBL. Atherosclerosis 2005; 178:107-113.

[7] Parhofer KG, Barrett PHR, Aguilar-Salinas CA, Schonfeld G. Positive linear correlation between the length of truncated apolipoprotein $B$ and its secretion rate: in vivo studies in human apoB-89, apoB-75, apoB-54.8, and apoB-31 heterozygotes. J Lipid Res 1996; 37:844852.

[8] Whitfield AJ, Barrett PHR, Robertson K, Havlat MF, van Bockxmeer FM, Burnett JR. Liver dysfunction and steatosis in familial hypobetalipoproteinemia. Clin Chem 2005; 51:266-269.

[9] Parhofer KG, Barrett PHR, Bier DM, Schonfeld G. Determination of kinetic parameters of apolipoprotein B metabolism using amino acids labeled with stable isotopes. J Lipid Res 1991; 32:1311-1323.

[10] Hooper AJ, Heeks L, Robertson K, et al. Lipoprotein metabolism in APOB L343V familial hypobetalipoproteinemia. J Clin Endocrinol Metab 2015; 100:E1484-1490.

[11] Hooper AJ, Robertson K, Barrett PH, Parhofer KG, van Bockxmeer FM, Burnett JR. Postprandial lipoprotein metabolism in familial hypobetalipoproteinemia. J Clin Endocrinol Metab 2007; 92:1474-1478.

[12] Ruotolo G, Zanelli T, Tettamanti C, et al. Hypobetalipoproteinemia associated with apo B-48.4, a truncated protein only 14 amino acids longer than apo B-48. Atherosclerosis 1998; 137:125131.

[13] Parhofer KG, Barrett PHR, Bier DM, Schonfeld G. Lipoproteins containing the truncated apolipoprotein, Apo B-89, are cleared from human plasma more rapidly than Apo B-100containing lipoproteins in vivo. J Clin Invest 1992; 89:1931-1937.

[14] Krul ES, Parhofer KG, Barrett PHR, Wagner RD, Schonfeld G. ApoB-75, a truncation of apolipoprotein B associated with familial hypobetalipoproteinemia: genetic and kinetic studies. J Lipid Res 1992; 33:1037-1050. 
Table 1. Summary of fasting lipids and VLDL-, IDL-, and LDL-apoB kinetics in controls and the apoB-80 FHBL subject.

\begin{tabular}{lcc}
\hline & Controls & ApoB-80 \\
& $\mathrm{n}=4$ & FHBL \\
\hline Age (years) & $24 \pm 1$ & 43 \\
Body mass index $\left(\mathrm{kg} / \mathrm{m}^{2}\right)$ & $20 \pm 0.5$ & 25 \\
Total cholesterol (mmol/L) & $3.7 \pm 0.25$ & 2.1 \\
Triglyceride (mmol/L) & $0.8 \pm 0.05$ & 0.4 \\
VLDL-apoB FCR (pools/d) & $8.4 \pm 0.85$ & 32.6 \\
VLDL-apoB production $(\mathrm{mg} / \mathrm{kg} / \mathrm{d})$ & $14.1 \pm 2.7$ & 13.0 \\
IDL-apoB FCR (pools/d) & $9.1 \pm 2.5$ & 15.3 \\
IDL-apoB production $(\mathrm{mg} / \mathrm{kg} / \mathrm{d})$ & $10.7 \pm 2.3$ & 4.6 \\
LDL-apoB FCR (pools/d) & $0.62 \pm 0.22$ & 0.76 \\
LDL-apoB production $(\mathrm{mg} / \mathrm{kg} / \mathrm{d})$ & $10.4 \pm 1.9$ & 3.2 \\
\hline
\end{tabular}

Data shown for controls are mean \pm SEM. 